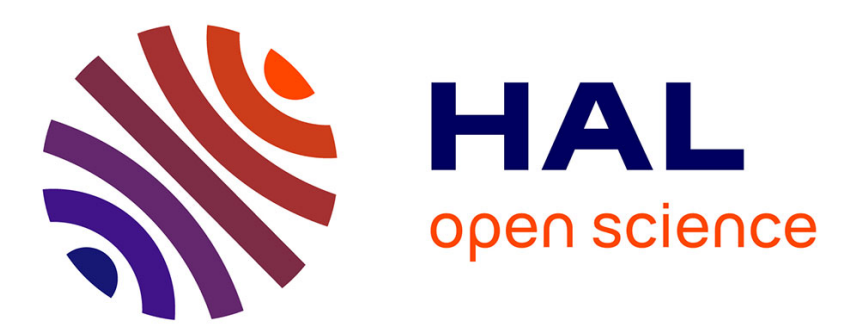

\title{
Hierarchical Coordinated Freeway On-Ramp Metering Using Switching System Theory
}

Antoine Lemarchand, John Jairo Martinez Molina, Damien Koenig

\section{To cite this version:}

Antoine Lemarchand, John Jairo Martinez Molina, Damien Koenig. Hierarchical Coordinated Freeway On-Ramp Metering Using Switching System Theory. SSSC 2010 - 4th IFAC Symposium on System, Structure and Control, Sep 2010, Ancona, Italy. hal-00544082

\section{HAL Id: hal-00544082 \\ https://hal.science/hal-00544082}

Submitted on 7 Dec 2010

HAL is a multi-disciplinary open access archive for the deposit and dissemination of scientific research documents, whether they are published or not. The documents may come from teaching and research institutions in France or abroad, or from public or private research centers.
L'archive ouverte pluridisciplinaire HAL, est destinée au dépôt et à la diffusion de documents scientifiques de niveau recherche, publiés ou non, émanant des établissements d'enseignement et de recherche français ou étrangers, des laboratoires publics ou privés. 


\title{
Hierarchical Coordinated Freeway On-Ramp Metering Using Switching System Theory
}

\author{
Antoine Lemarchand* John J. Martinez* Damien Koenig * \\ * Control system department, GIPSA-lab, UMR 5216, Grenoble-INP, \\ France.
}

\begin{abstract}
This paper deals with the problem of freeway traffic control through ramp metering to enhance their efficiency. The traffic is often modeled by a nonlinear hyperbolic conservation laws that may develop and propagate shock waves. The Cell Transmission model (CTM) developed in (Daganzo [1994]),(C. F. Daganzo [1995]) is taken in this work since linear control tools can be used for analysis and control design. This model is extended with parametric uncertainties. A hierarchical control scheme with two levels is considered. The first level gives the optimal densities to apply on the freeway. The second level is charged for compensating local parametric uncertainties and disturbances (on-ramp demands, boundary conditions). These controllers are designed using switching control theory. A real-data based simulation is used to validate the effectiveness of the proposed control approach.
\end{abstract}

Keywords: Traffic control, switching theory, linear optimal control, uncertain linear systems

\section{INTRODUCTION}

According to the 2007 urban mobility report (Schrank and Lomax [2007]), the cost of traffic jam has increased $5 \%$ during the last three years and has reached more than 4.2 billion dollars. This is due to the increasing amount of vehicles on roads that also causes an over consumption of fuel. Since the construction of new roads may be very expensive and not always possible, efforts trying to optimize the use of the existing road network are reinforced. A valuable tool for increasing the effectiveness of the design and the evaluation of traffic monitoring and control is the use of accurate traffic models (Munoz et al. [2004]). There are two types of traffic models : microscopic and macroscopic models. In microscopic models, each cars behavior is considered separately (Yang and Koutsopoulos [1996]) whereas in macroscopic models density and flow are considered as in hydrodynamic theory (Daganzo [1994]). In this work, we choose a first order macroscopic model which can not predict negative flow, unlike higher order models (Papageorgiou [1998]). First order models are usually based on the Cell Transmission Model (CTM) presented in (C. F. Daganzo [1995]). The main idea of these models is to consider homogenous density over small interconnected road sections and to calculate flow between cells in case of simple, merging and diverging junctions (Section 2). The CTM is extended in order to propose a new uncertain multiple state space representation. This work is presented in Section 3. In this paper we use a hierarchical control scheme in order to achieve a global control of a freeway section which include several on/offramps. This scheme is depicted in Figure 1. Here, the control system is divided in two layers, a high level layer which determines the optimal traffic densities and flows as in (Gomes and Horowitz [2006]),(Jacquet [2008]). The solution of the optimal problem is computed by the simplex algorithm. This layer is presented in section 4 . Once the optimal references are calculated by this layer, they are applied at each on-ramp neighborhood. Due to the presence of disturbances, model uncertainties and the multi-model, a bank of local PI controllers based on the Lyapunov theory on switching systems is designed via LMI formulation as explored in (Jacquet et al. [2008]). This work is presented in Section 5. Finally, a real-data based simulation is performed to illustrate the performance of the proposed control strategy. This work is presented in Section 6 .

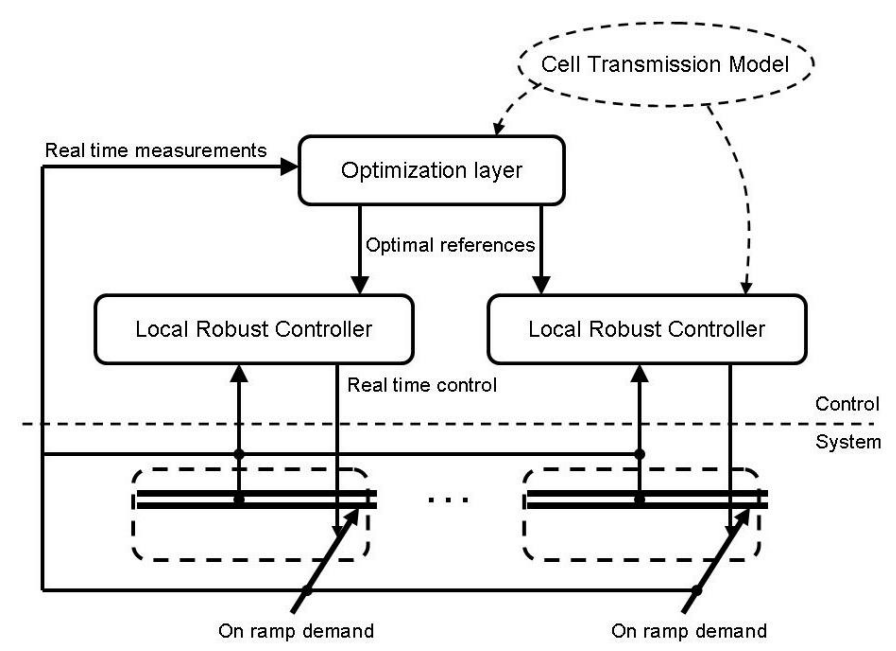

Fig. 1. Regulation scheme.

\section{CELL TRANSMISSION MODEL}

The CTM is a first order linear discrete traffic model proposed in (Daganzo [1994]),(C. F. Daganzo [1995]) and used 
in many trafic issues. The CTM model is presented in four parts : Section 2.1 presents the elementary cells, Section 2.2 presents the queue model, Section 2.3 presents the junction model, and Section 2.4 presents the uncertainties model.

\subsection{Elementary Cell}

Consider the road section divided into elementary cells. Assuming that in each cell, the density, $\rho(k)[\mathrm{veh} / \mathrm{km}]$, of vehicles is homogenous. Then $\rho(k)$ can be related to $\phi_{i}(k)$ and $\phi_{i+1}(k)[\mathrm{veh} / \mathrm{h}]$, respectively the flow entering and leaving cell $i$ by

$$
\rho_{i}(k+1)=\rho_{i}(k)+\frac{T}{l_{i}}\left(\phi_{i}(k)-\phi_{i+1}(k)\right),
$$

as depicted in Figure 2

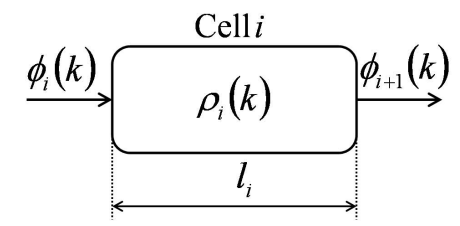

Fig. 2. Elementary cell.

where $l_{i}[\mathrm{~km}]$ is the length of cell $i$ and $T[\mathrm{~h}]$ the period of discretized time. To guarantee numerical stability, $T$ has to fit the condition $T<\inf \left(\frac{l_{i}}{v_{i}}\right), \forall i=1, \ldots, N$ ( $N$, the number of cells). Notice that (1) is the conservation law of vehicles (Jacquet [2007]).

\subsection{Queue}

The queue model is the same as the cell model (section 2.1). Taking $d_{i}(k)[\mathrm{veh} / \mathrm{h}]$ as the demand at on-ramp (i.e. the flow entering on-ramp), and $u_{i}(k)[\mathrm{veh} / \mathrm{h}]$ as the flow leaving on-ramp and entering in the main line (Figure 3 ). The evolution of the queue density, $\rho_{i n_{i}}(k)[\mathrm{veh} / \mathrm{km}]$ is described by

$$
\rho_{i n_{i}}(k+1)=\rho_{i n_{i}}(k)+\frac{T}{l_{i n_{i}}}\left(d_{i}(k)-u_{i}(k)\right),
$$

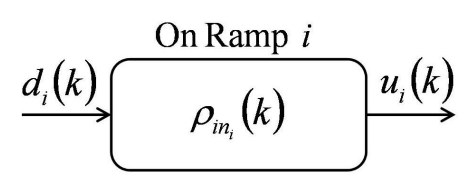

Fig. 3. Queue.

\subsection{Junctions}

Assuming that a cell can contain at most one on-ramp at the beginning of the cell and one off-ramp at the end as depicted in Figure 4. Denote $u_{i}$ the flow entering cell $i$ via on-ramp, and $\phi_{\text {out }_{i}}$ the flow leaving cell $i-1$ via off-ramp.

Define $S_{\phi_{i}}(k)$ as the flow that the upstream cell can supply, and $R_{\phi_{i}}(k)$ as the flow that the downstream cell can receive. They depend on $v_{i}$ the free flow speed, $w_{i}$ the backward congestion propagation speed, $\rho_{J_{i}}$ the jam

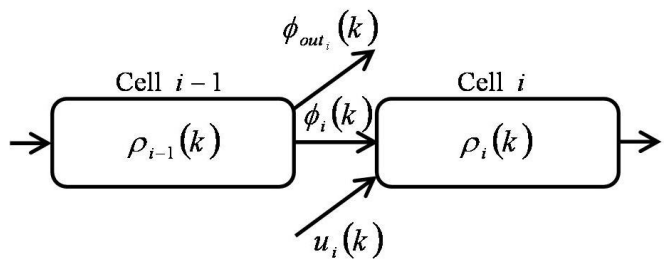

Fig. 4. Junction.

density (i.e. maximal density), and $\phi_{M_{i}}$ the maximum flow that can travel from upstream to downstream cell. $S_{\phi_{i}}(k)$ and $R_{\phi_{i}}(k)$ are characterizeds by :

$$
\begin{aligned}
& S_{\phi_{i}}(k)=\min \left(\left(1-\beta_{i}\right) \cdot v_{i-1} \cdot \rho_{i-1}(k), \phi_{M_{i}}\right), \\
& R_{\phi_{i}}(k)=\min \left(w_{i} \cdot\left(\rho_{J_{i}}-\rho_{i}(k)\right)-\phi_{i n_{i}}(k), \phi_{M_{i}}\right) .
\end{aligned}
$$

with $\beta_{i}$ the split ratio (i.e. the part of the flow leaving cell $i-1$ that leaves main road via off-ramp) defined by

$$
\phi_{\text {out }_{i}}(k)=\beta_{i} \cdot\left(\phi_{\text {out }_{i}}(k)+\phi_{i}(k)\right) .
$$

Obviously the flow moving from upstream to downstream cell is the minimum of (3) and (4), i.e.

$$
\phi_{i}(k)=\min \left(S_{\phi_{i}}(k), R_{\phi_{i}}(k)\right) .
$$

Junction modes :

From (3), (4), and (6) one can identify the three possible modes of the junction : a free mode $(\mathrm{F})$ where the flow is proportional to the upstream cell concentration, a decoupled mode (D) where the flow is equal to the maximal flow, and a congested mode $(\mathrm{C})$ where the flow is proportional to the remaining space in downstream cell. A graphical representation of (3), (4) and (6) is provided in Figure 5. It is called the fundamental diagram. This kind of diagram appears in every traffic issues using macroscopic model (Geroliminis and Daganzo [2008]).

\subsection{Uncertain Parameters}

The nominal parameters of the fundamental diagram can be computed using the calibration methods described in (Munoz et al. [2004]) with experimental data ${ }^{1}$. The obtained diagram is depicted in Figure 5.

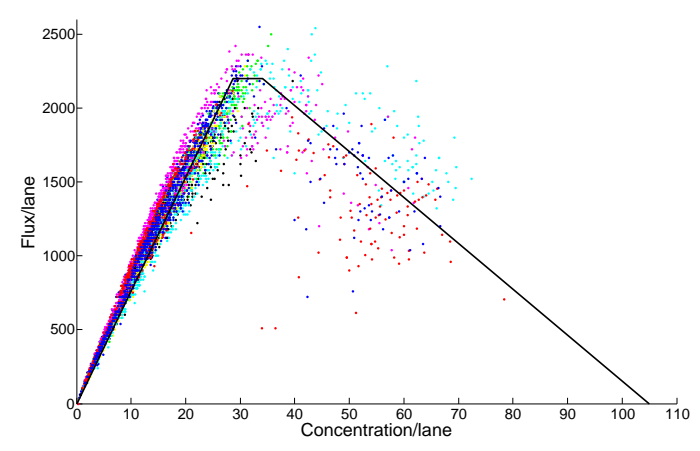

Fig. 5. Fundamental diagram identification.

1 Real time measurements realized on D383 road (near Lyon, France) provided by DDE69 
Notice that this diagram contains uncertainties. They can be modeled as the following parametric uncertainties ( $\mathrm{Cf}$ Figure 6) :

$$
\begin{aligned}
v_{i-1}(k) & =v_{0_{i-1}}+\Delta v_{i-1}(k) \\
\phi_{M_{i}}(k) & =\phi_{M_{0_{i}}}+\Delta \phi_{M_{i}}(k) \\
w_{i}(k) & =w_{0_{i}}+\Delta w_{i}(k)
\end{aligned}
$$

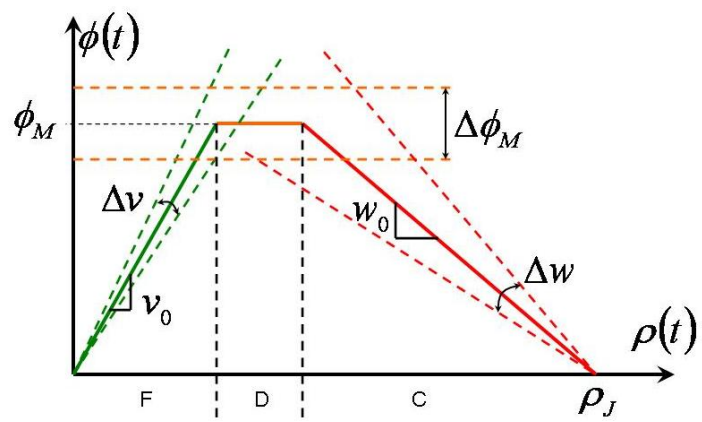

Fig. 6. Fundamental diagram with parametric uncertainties.

The spectrum of $\Delta v$ is depicted in Figure 7 . It shows that this parameter has a slow varying rate. Indeed, the most important part of the uncertainty is situated at low frequencies (less than $0.5 h^{-1}$ ). So a local bank of PI controller can attenuate these uncertainties.

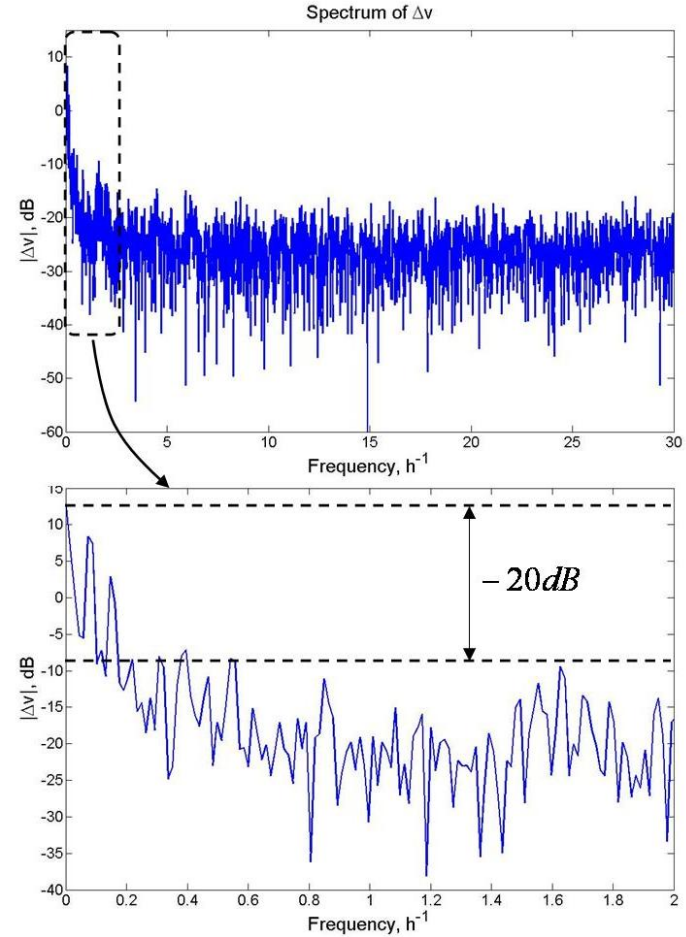

Fig. 7. Spectrum of $\Delta v$.

\section{UNCERTAIN SWITCHED CTM}

Consider a freeway section, divided into $N$ cells, $N+1$ junctions and $N_{\text {in }}$ inputs. Define

$$
\alpha(k):=\left[\alpha_{1}(k), \cdots, \alpha_{N+1}(k)\right]
$$

where $\alpha_{i}(k) \in\{F, D, C\}$ represents the mode of junction $i$ (see Section 2.3).
Proposition 1. For all $\alpha(k)$ the dynamics of a freeway section can be written as a dynamical system of the form

$$
\begin{array}{r}
\rho(k+1)=A_{0_{\alpha(k)}} \cdot \rho(k)+B_{\alpha(k)} \cdot u(k)+E_{\alpha(k)} \cdot d(k)+ \\
a_{0_{\alpha(k)}}+F_{\alpha(k)} \cdot \rho_{\Delta}(k)+G_{\alpha(k)} \cdot h_{\Delta}(k)
\end{array}
$$

where $\rho(k)$ is the state vector of densities in each cells, $u(k)$ are controlled on-ramps flows, $d(k)$ are uncontrolled on-ramps flows and boundary conditions, $a_{0_{\alpha(k)}}$ is a constant, $\rho_{\Delta}(k)$ and $h_{\Delta}(k)$ are some perturbations. $A_{0_{\alpha(k)}}, B_{\alpha(k)}, E_{\alpha(k)}, F_{\alpha(k)}, G_{\alpha(k)}$ are matrices of appropriate dimensions.

Proof In the rest of this section, we will write $\alpha$ instead of $\alpha(k)$ on clearness purpose.

Firstly, the conservation law (1) is rewritten as follows :

$$
\rho(k+1)=\rho(k)+\Theta . \phi(k)+B . u(k)
$$

where $\phi=\left[\phi_{1}, \cdots, \phi_{N+1}\right]$ is the vector of flows in each junction, and

$$
\begin{gathered}
\Theta=\left(\begin{array}{cccc}
\frac{T}{l_{1}} & -\frac{T}{l_{1}} & 0 & 0 \\
0 & \ddots & \ddots & 0 \\
0 & 0 & \frac{T}{l_{N}} & -\frac{T}{l_{N}}
\end{array}\right), \\
B=\left(\begin{array}{ccc}
0 & 0 & 0 \\
\frac{T}{l_{i 1}} & 0 & \vdots \\
0 & \frac{T}{l_{i x}} & \vdots \\
\vdots & 0 & 0 \\
0 & 0 & \frac{T}{l_{i N_{i n}}}
\end{array}\right) .
\end{gathered}
$$

Secondly, the vector $\phi(k)$ is reformulated thanks to the results of Section 2.3 with a matrix formulation as follows

$$
\begin{gathered}
\phi(k)=A_{\phi_{\alpha}}(k) \cdot \rho(k)+B_{\phi_{\alpha}} \cdot u(k)+E_{\phi_{\alpha}} \cdot d(k) \\
+a_{J_{\alpha}}(k) \cdot \rho_{J}+a_{\phi_{M \alpha}} \cdot \phi_{M}(k),
\end{gathered}
$$

where, $\rho_{J}:=\left[\rho_{J_{1}}, \cdots, \rho_{J_{N}}\right]$ is the vector jam densities of each cell, $\phi_{M}(k):=\left[\phi_{M_{1}}(k), \cdots, \phi_{M_{N+1}}(k)\right]$ is the vector of maximal flows in each junction (see Figure 6), and the matrices $A_{\phi_{\alpha}}(k), B_{\phi_{\alpha}}, E_{\phi_{\alpha}}, a_{J_{\alpha}}(k), a_{\phi_{M \alpha}}$ are respectively described by :

$$
A_{\phi_{\alpha}}(k)=\left(\begin{array}{ccc}
-w_{1}(k) \cdot e_{C_{1}} & 0 & 0 \\
v_{1}(k) \cdot e_{F_{2}} & \ddots & 0 \\
0 & \ddots & -w_{N}(k) \cdot e_{C_{N}} \\
0 & 0 & v_{N}(k) \cdot e_{F_{N+1}}
\end{array}\right),
$$

$$
B_{\phi_{\alpha}}=\left(\begin{array}{ccc}
0 & 0 & 0 \\
-e_{C_{1}} & 0 & \vdots \\
0 & -e_{C_{x}} & \vdots \\
\vdots & 0 & 0 \\
0 & 0 & -e_{C_{N i n}}
\end{array}\right) \text {, }
$$




$$
\begin{gathered}
E_{\phi_{\alpha}}=\left(\begin{array}{cc}
e_{F_{1}} & 0 \\
0 & \vdots \\
\vdots & 0 \\
0 & e_{C_{N+1}}
\end{array}\right), \\
a_{J_{\alpha}}(k)=\left(\begin{array}{ccc}
w_{1}(k) & & 0 \\
& \ddots & \\
0 & & w_{N+1}(k)
\end{array}\right), \\
a_{\phi_{M \alpha}}=\left(\begin{array}{ccc}
e_{D_{1}} & & 0 \\
& \ddots & \\
0 & & e_{D_{N}}
\end{array}\right),
\end{gathered}
$$

with,

$$
\begin{aligned}
e_{F_{i}} & = \begin{cases}1 & \text { if junction } i \text { is Free } \\
0 & \text { otherwise }\end{cases} \\
e_{D_{i}} & = \begin{cases}1 & \text { if junction } i \text { is Decoupled } \\
0 & \text { otherwise }\end{cases} \\
e_{C_{i}} & = \begin{cases}1 & \text { if junction } i \text { is Congested } \\
0 & \text { otherwise }\end{cases}
\end{aligned}
$$

Substituting (10) into (9) and using the relations (11-14) the formulation (15) is obtained.

$$
\begin{aligned}
A_{\alpha}(k) & =I+\Theta \cdot A_{\phi_{\alpha}}(k), \\
B_{\alpha} & =B+\Theta \cdot B_{\phi_{\alpha}}, \\
E_{\alpha} & =\Theta \cdot E_{\phi_{\alpha}}, \\
a_{\alpha}(k) & =\Theta \cdot\left(a_{J_{\alpha}}(k) \cdot \rho_{J}+a_{\phi_{M \alpha}} \cdot \phi_{M}(k)\right) .
\end{aligned}
$$

$$
\rho(k+1)=A_{\alpha}(k) \cdot \rho(k)+B_{\alpha} \cdot u(k)+E_{\alpha} \cdot d(k)+a_{\alpha}(k)(.15)
$$

Notice that (11) and (14) contain uncertainties. Let's separate the constant parts and the uncertain parts of (11) and (14). For this purpose, we study a case where all different junction modes occurs : the freeway section is in congested mode from junction 1 to $p-1$, decoupled in junction $p$ and free from $p+1$ to $N+1$. From (7) the term (11) can be decomposed as a nominal and an uncertain part as follows :

$$
A_{\alpha}(k)=A_{0_{\alpha}}+F_{\alpha} \cdot \Delta(k)
$$

with,

$$
\begin{aligned}
& A_{0_{\alpha}}=\left.A_{\alpha}(k)\right|_{v_{i}(k)=v_{0_{i}}, w_{i}(k)=w_{0_{i}}}, \\
& F_{\alpha}=\Theta .\left(\begin{array}{ccccc}
1 & & & & 0 \\
& \ddots & & & \\
& & 1 & & \\
& & 1 & & \\
& & & \ddots & \\
0 & & & & 1
\end{array}\right) \\
& \Delta(k)=\left(\begin{array}{cccccc}
\Delta w_{1}(k) & & & & & \\
& \ddots & & & 0 & \\
& & \Delta w_{p-1}(k) & & & \\
& & & \Delta v_{p}(k) & & \\
& 0 & & & \ddots & \\
& & & & & \Delta v_{N}(k)
\end{array}\right)
\end{aligned}
$$

The term (14) can be decomposed as :

with

$$
a_{\alpha}(k)=a_{0_{\alpha}}+G_{\alpha} \cdot h_{\Delta}(k)
$$

$$
\begin{aligned}
& a_{0_{\alpha}}=\left.a_{\alpha}(k)\right|_{w_{i}(k)=w_{0_{i}}, \phi_{M_{i}}(k)=\phi_{M_{0_{i}}}}, \\
& G_{\alpha}=\Theta \cdot\left(\begin{array}{lll}
1 & & 0 \\
& \ddots & \\
0 & & 1 \\
& &
\end{array}\right) \text {, } \\
& h_{\Delta}(k)=\left(\begin{array}{c}
\rho_{J_{1}} \cdot \Delta w_{1}(k) \\
\vdots \\
\rho_{J_{p-1}} \cdot \Delta w_{p-1}(k) \\
\Delta \phi_{M_{p}}
\end{array}\right),
\end{aligned}
$$

Substituting (16) and (17) in (15), and taking $\rho_{\Delta}(k)=$ $\rho(k) . \Delta(k)$ the formulation (8) of the proposition is obtained.

\section{OPTIMIZATION LAYER}

The optimization problem is a non-linear and non-convex problem. This type of problem is hard to solve and we can not guarantee that the optimal solution found is the global optimal solution. To solve this problem, the optimization problem has to be converted into a linear and convex problem. This conversion is proposed and demonstrated in (Gomes and Horowitz [2006]),(Jacquet [2008]).

We denote $\chi$ the set of cells, $\zeta$ those which contain an offramp, and $\xi$ those which contain an on-ramp. Assume that each on-ramp can be metered. Consider the time horizon $t \in[0,(K-1) \cdot T] \Leftrightarrow k \in \kappa=[0, K-1]$ to compute the optimization, with $T$ the sampling time.

\subsection{Constraints}

The first part of the constraints of the linear problem consists in the main-line and on-ramp conservation laws, respectively (18) and (19).

$$
\begin{aligned}
& \left\{\begin{array}{c}
\rho_{i}(k+1)=\rho_{i}(k)+\frac{T}{l_{i}}\left(\phi_{i}(k)-\phi_{i+1}(k)\right. \\
\quad \forall i \in \chi, \forall k \in \kappa
\end{array}\right. \\
& \left\{\begin{array}{l}
\rho_{i n_{i}}(k+1)=\rho_{i n_{i}}(k)+\frac{T}{l_{i n_{i}}}\left(d_{i}(k)-u_{i}(k)\right) . \\
\forall i \in \xi, \forall k \in \kappa
\end{array}\right.
\end{aligned}
$$

Notice that in (18), $\phi_{i n_{i}}(k)=0$ if cell $i \notin \xi$, and $\phi_{\text {out }_{i}}(k)=0$ if $i \notin \zeta$. To ensure that the concentration and flow does not become negative and does not exceed maximum capabilities, constraints on the on-ramp flow and concentration are implemented as follows :

$$
\left\{\begin{array}{l}
\rho_{i n_{i}}(k) \leq \rho_{\operatorname{Min}_{i}}, \\
\phi_{i n_{i}}(k) \leq \frac{l_{i n_{i}}}{T} u_{i}(k), \\
\phi_{i n_{i}}(k) \leq \phi_{\operatorname{Min}_{i}}(k), \\
\forall i \in \xi, \forall k \in \kappa
\end{array}\right.
$$

The fundamental diagram which allows us to calculate the flow in the junction is a non-linear function due to the $\mathrm{min}$ 
function in (3) and (4). A well known trick of relaxation of such a function is to replace this function by a set of upper bounds and to add the relaxed variable on the criteria with appropriate optimization direction (25) as done in (Gomes and Horowitz [2006]). So $\forall i \in \chi$ and $\forall k \in \kappa$ the non-linear characteristic (6) can be replaced by

$$
\left\{\begin{array}{l}
\phi_{i}(k) \leq\left(1-\beta_{i-1}\right) \cdot v_{i-1} \cdot \rho_{i-1}(k) \\
\phi_{i}(k)+\phi_{i n_{i}}(k) \leq w_{i} \cdot\left(\rho_{J_{i}}-\rho_{i}(k)\right) \\
\phi_{i}(k) \leq \phi_{M_{i}} \\
\forall i \in \chi, \forall k \in \kappa
\end{array}\right.
$$

\subsection{Criteria}

The criteria is divided into three parts. The Total Travel Time (TTT) represents the time spend by all vehicle traveling on the main section. The Total Waiting Time (TWT) represents the time that vehicles spend waiting at on-ramps. And the Total Travel Distance (TTD) represents the total distance traveled by all vehicles. The TTT and the TWT has to be minimized. The TTD has to be maximized to fit the previous relaxation. So the criteria $J$ to be minimized is

with,

$$
J=T T T+\mu T W T-\eta T T D
$$

$$
\begin{aligned}
T W T & =\sum^{\kappa} \sum_{\chi}^{\xi} \rho_{i n_{i}}(k) . l_{i n_{i}}, \\
T T T & =\sum^{\kappa} \sum^{\kappa} \rho_{i}(k) . l_{i}, \\
T T D & =\sum^{\kappa}\left(\sum^{\chi} \phi_{i}(k)+\sum^{\xi} u_{i}(k)\right) .
\end{aligned}
$$

\subsection{Linear Problem}

So, the optimal references $\left(\rho^{*}(k), u^{*}(k)\right)$, are the solution of the linear problem optimization :

$$
\left\{\begin{array}{rl}
\min _{\rho, u} & J \\
\text { subject to } & (18),(19), \text { conservation equations } \\
& (21), \text { main line constraints } \\
& (20), \text { on-ramp constraints }
\end{array}\right.
$$

This problem is solved using the GLPK (GNU Linear Programming Kit) library which implements the Simplex algorithm.

\section{LOCAL REGULATION}

The optimal trajectories $\left(\rho^{*}(k), u^{*}(k)\right)$ calculated with the optimization layer are open loop calculations. Nominal system models with estimated parameters is used. To ensure that the system track the references, we design a local regulation at each neighborhood of metered on-ramp as it is shown in Figure 8.

\subsection{Case Study}

Take the neighborhood depicted in Figure 9. Assume that junctions 5 and 1 are always in Free mode.

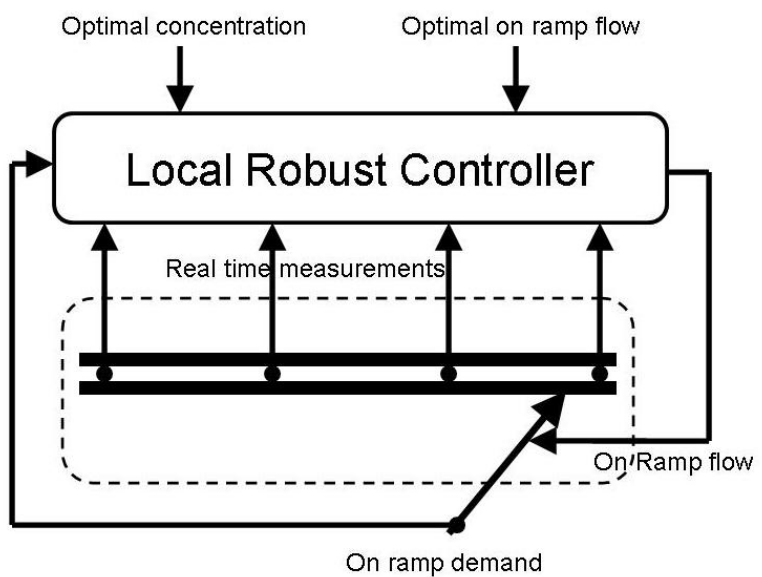

Fig. 8. Local regulation.

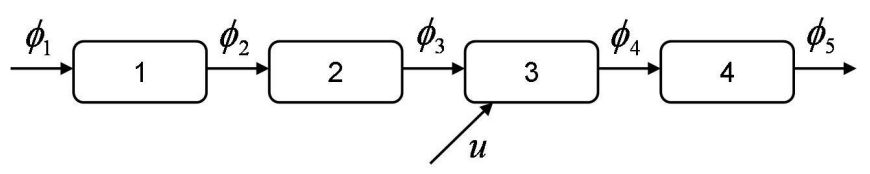

Fig. 9. On-ramp neighborhood.

In normal traffic conditions (i.e. without accidents) the different modes which a system can experience are the following :

- case $1: \alpha(k)=[F F F F F]$

- case $2: \alpha(k)=[F F F D F]$

- case $3: \alpha(k)=[F F C D F]$

- case $4: \alpha(k)=[F C C D F]$

In case 1 , all the junctions of the section are in free mode. Just before congestion appears (case 2) junction 4 switches to decoupled mode. Then in case 3 and 4 , the congestion propagates backward. For theses 4 cases, we study the controllability of the system, with the Kalman criteria as in Munoz et al. [2003]. In mode 1, the densities of cells 3 and 4 are controllable. In mode 2 , the density of cell 3 is controllable. In mode 3 , the density of cell 2 is controllable. In mode 4 , the densities of cells 1 and 2 are controllable. Denoting the transition from case 1 to case 2 as $\tau_{12}$. We can write the possible transition set :

$$
\Gamma=\left\{\tau_{11}, \tau_{12}, \tau_{21}, \tau_{22}, \tau_{23}, \tau_{32}, \tau_{33}, \tau_{34}, \tau_{43}, \tau_{44}\right\} .
$$

\subsection{Extended System}

Since the optimization layer is developed with the nominal system (i.e. without uncertain parameters) and based on the results of Section 4 , the optimal references $\left(\rho^{*}(k), u^{*}(k)\right)$ respect

$\rho^{*}(k+1)=A_{0_{\alpha(k)}} \cdot \rho^{*}(k)+B_{\alpha(k)} \cdot u^{*}(k)+E_{\alpha(k)} \cdot d(k)+a_{0_{\alpha(k)}}$.

Take

$$
\begin{aligned}
& \epsilon(k)=\rho(k)-\rho^{*}(k), \\
& v(k)=u(k)-u^{*}(k),
\end{aligned}
$$

then from (8) and (28), we can write 


$$
\begin{aligned}
\epsilon(k+1)=A_{0_{\alpha(k)}} \cdot \epsilon(k) & +B_{\alpha(k)} \cdot v(k)+F_{\alpha(k)} \cdot \rho_{\Delta}(k) \\
& +G_{\alpha(k)} \cdot h_{\Delta}(k),
\end{aligned}
$$

The problem now, is to design a robust switched PI controller in order to stabilize the system (29) face to the uncertainties $\rho_{\Delta}(k), h_{\Delta}(k)$. For this purpose, we have to extend our system with an integrator. The new state vector becomes

$$
\epsilon_{a}(k)=\left(\begin{array}{c}
\epsilon(k) \\
z(k)
\end{array}\right)
$$

To keep the stabilizability property of the system, we can only extend one state of the system with an integrator. For the cases 1 and 2, we choose to extend the system with an integrator on $\epsilon_{3}(k)$. For the cases 3 and 4 , we choose to extend the system with an integrator on the cell density where the congestion front stand (respectively $\epsilon_{2}(k)$ and $\left.\epsilon_{1}(k)\right)$. So,

$$
z(k+1)=z(k)+ \begin{cases}\epsilon_{3}(k) & , \text { if case } 1 \text { or } 2 \\ \epsilon_{2}(k) & , \text { if case } 3 \\ \epsilon_{1}(k) & , \text { if case } 4\end{cases}
$$

The nominal extended system is described by :

$$
\epsilon_{a}(k+1)=A_{a_{\alpha}} \cdot \epsilon(k)+B_{a_{\alpha}} \cdot v(k),
$$

with the following dynamics matrices:

$$
\begin{aligned}
A_{a_{\alpha}} & =\left(\begin{array}{ll}
A_{0_{\alpha(k)}} & 0 \\
A_{z_{\alpha(k)}} & 1
\end{array}\right), \\
B_{a_{\alpha}} & =\left(\begin{array}{c}
B_{\alpha(k)} \\
0
\end{array}\right),
\end{aligned}
$$

According to 31, we have

$$
A_{z_{\alpha(k)}}= \begin{cases}\left(\begin{array}{llll}
0 & 0 & 1 & 0
\end{array}\right) & , \text { if case } 1 \text { or } 2 \\
\left(\begin{array}{llll}
0 & 1 & 0 & 0
\end{array}\right) & , \text { if case } 3 \\
\left(\begin{array}{llll}
1 & 0 & 0 & 0
\end{array}\right) & , \text { if case } 4\end{cases}
$$

\subsection{State Feedback Design}

Assume that each cell density $\rho_{i}, i \in 1, \ldots, 4$ is measured. This seems to be a fair assumption since sensor becomes cheaper and cheaper, and since many roads are equipped with sensors. Then, we apply the following state feedback on the system (32)

$$
u(k)=-K_{\alpha(k)} \epsilon_{a}(k) .
$$

Take the following notations, $\alpha(k)=i, \alpha(k+1)=j$

The core of our approach is the use of the following candidate Lyapunov function

$$
V_{i}(k)=\epsilon_{a}(k)^{T} P_{i} \epsilon_{a}(k) .
$$

Where $P_{i}$ is a positive definite matrix. Since $P_{i}=P_{i}^{T}>0$, it is well known that $\Delta V(k)=V_{j}(k+1)-V_{i}(k)<0$ is a sufficient condition to prove the stability of the system. As,

$$
\Delta V(k)=\epsilon_{a}(k+1)^{T} P_{j} \epsilon_{a}(k+1)-\epsilon_{a}(k)^{T} P_{i} \epsilon_{a}(k)
$$

$=\epsilon_{a}(k)^{T}\left(\left(A_{a_{i}}-B_{a_{i}} K_{i}\right)^{T} P_{j}\left(A_{a_{i}}-B_{a_{i}} K_{i}\right)-P_{i}\right) \epsilon_{a}(k)$, the system is stable under the state feedback (33) if

$$
\left(A_{a_{i}}-B_{a_{i}} K_{i}\right)^{T} P_{j}\left(A_{a_{i}}-B_{a_{i}} K_{i}\right)-P_{i}<0 .
$$

(37) is a BMI that can easily be rewritten as an LMI. Take $Q_{i}=P_{i}^{-1}, X_{i}=K_{i} P_{i}^{-1}$ and multiply (37) on the left and on the right by $P_{i}^{-1}$. So (37) becomes

$$
\left(A_{a_{i}} Q_{i}-B_{a_{i}} X_{i}\right)^{T} Q_{j}^{-1}\left(A_{a_{i}} Q_{i}-B_{a_{i}} X_{i}\right)-Q_{i}<0
$$

Using the Schur's complements, (38) can be written as follows :

$$
\left(\begin{array}{cc}
Q_{j} & \left(A_{a_{i}} Q_{i}-B_{a_{i}} X_{i}\right) \\
* & Q_{i}
\end{array}\right)>0 .
$$

As in (Montagner et al. [2003]), this condition can be modified to place the poles of the system in a particular region as depicted in Figure 10.

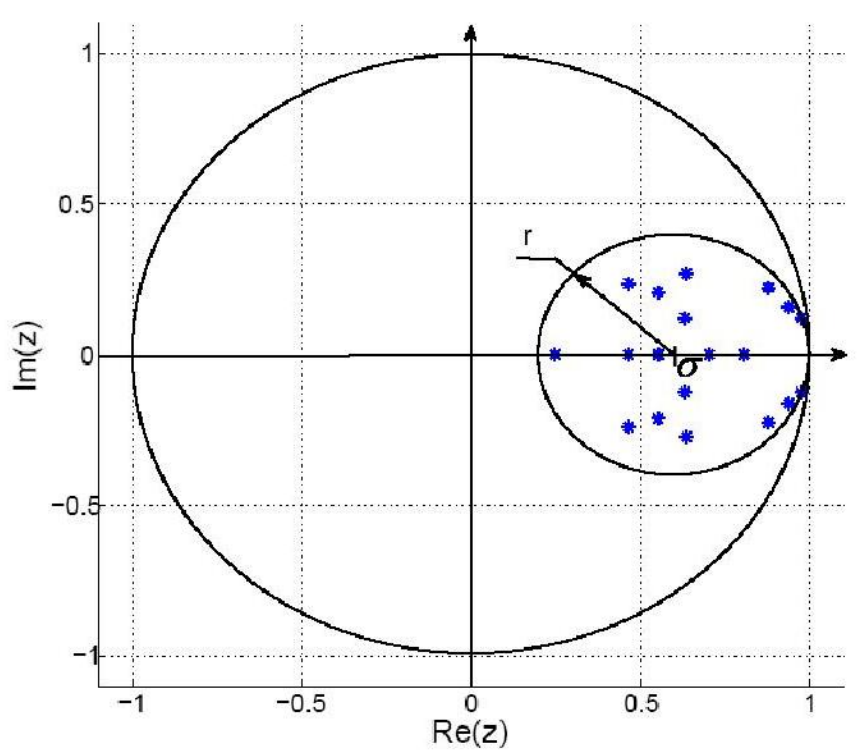

Fig. 10. Pole placement region.

For this purpose, we have to replace $A_{i}-B_{i} K_{i}$ by $\frac{A_{i}-B_{i} K_{i}-(1-r) I}{r}$. So the condition (39) becomes :

$$
\left(\begin{array}{cc}
r Q_{j} & A_{a_{i}} Q_{i}-B_{a_{i}} X_{i}-(1-r) Q_{i} \\
* & r Q_{i}
\end{array}\right)>0 .
$$

The LMI condition (40), gives us a sufficient condition of stability and pole placement for the extended system. From here, we can have two approaches. We can take a single Lyapunov function and a single state feedback for all $\alpha(k)$ (i.e. $P_{i}=P_{j}=P$ and $K_{i}=K_{j}=K, \forall i, j$ ). Or we can take a different Lyapunov function and state feedback for each suitable value of $\alpha(k)$. Obviously, the approach with a single regulator is more conservative and the solution may not exist for particular placement region. If this solution exists, the controller is easier to implement. The solution of the LMI (39) gives the local gain $K=X . P$. For the multiple controller approach, the LMI (39) has to be satisfied for all transitions of $\Gamma(27)$.

Figure 11 shows the sensitivity function between relevant uncertainties $\left(\Delta v_{1}, \Delta w_{3}, \Delta \phi_{M}\right)$ and the extended output $\epsilon_{3}$ in mode 3 . As we can see, the influence of uncertainties is attenuated at low frequency.

\section{REAL-DATA BASED SIMULATION}

The control law is tested with real traffic measurement of a section of the D383 road near Lyon, France, source : DDE69 (Direction departementale de l'equipement Rhone 


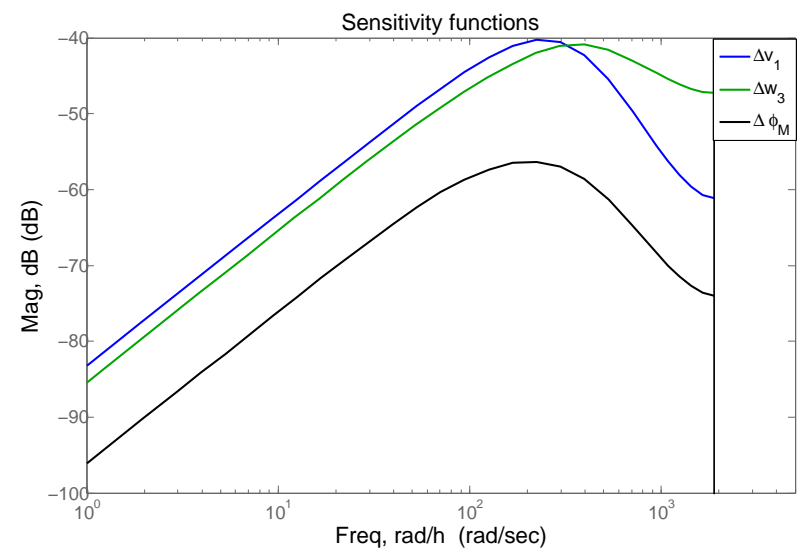

Fig. 11. Sensitivity functions.

Alpes). This section and the measurement points are represented on Figure 12, and has been segmented as shown on Figure 13. The period of time selected correspond to the after work congestion which is a critical moment for traffic management.

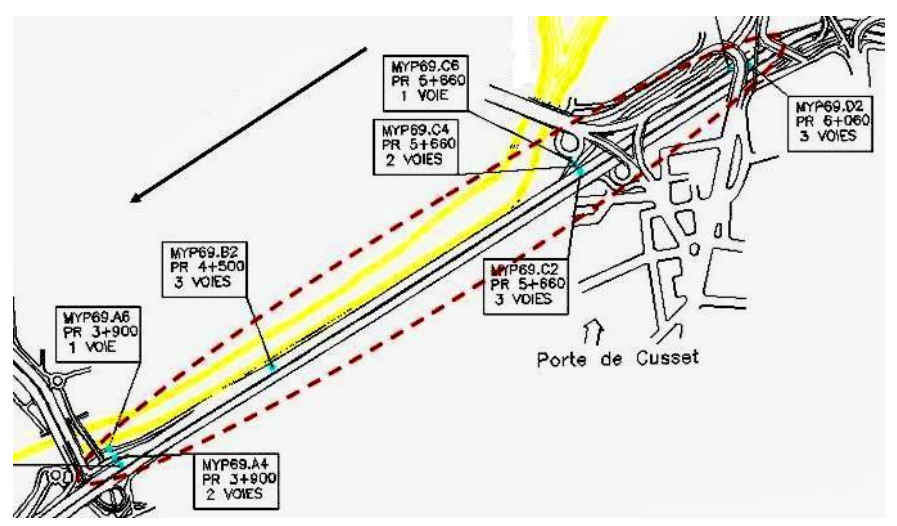

Fig. 12. Application freeway section D383.

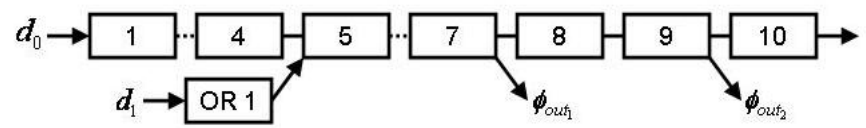

Fig. 13. Application freeway section/segmentation.

The system is simulated thanks to the model presented in Section 3, subject to the uncertainties depicted in Figure 14 .

For the synthesis of the local controllers, we consider a neighborhood constituted by cell 1 to 6 of the section depicted in Figure 13. The result of pole placement is depicted in Figure (10).

Figure 15 shows the evolution of densities and the on-ramp density subject to the optimal on-ramp flow (computed by optimization layer) without local controllers. There are two critical periods (around 1.5 and 2.5) where the system is perturbed by uncertainties. Notice that the uncertainty $\Delta \phi_{M}$ has more effect on the system.

Figure 16 shows the evolution of densities subject to the optimal on-ramp flow with local controllers. The graph call "Active Controller" . To avoid useless queue, the

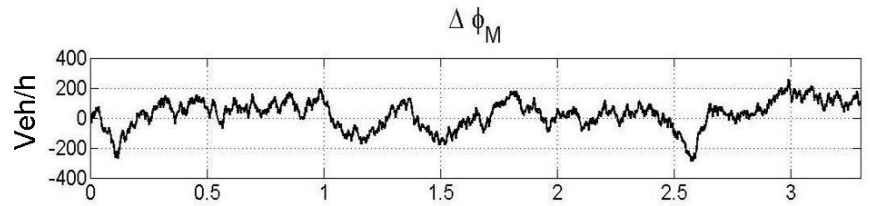

$\Delta \mathrm{V}$

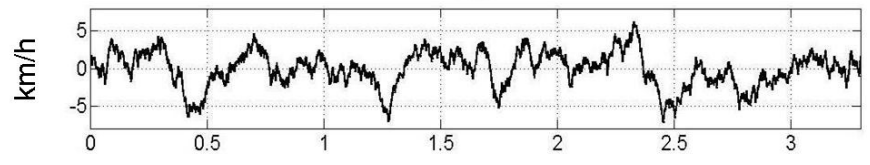

$\Delta w$

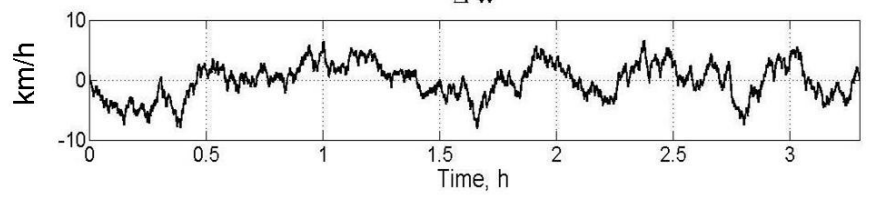

Fig. 14. Uncertainties.
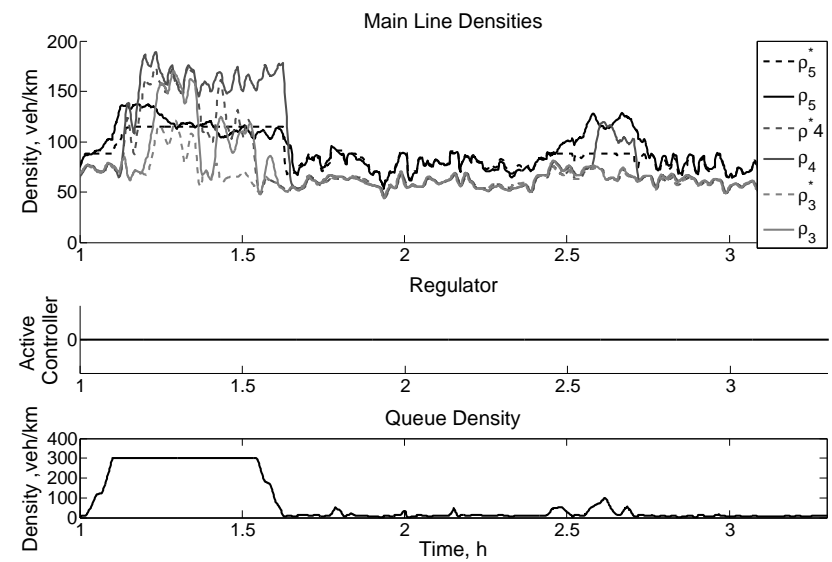

Fig. 15. Optimal command.

case 1 is un-metered. The effect of uncertainties on the evolution of the system is well attenuated. The controllable densities track optimal references. Here the queue exceeds maximum capacity which is 300 .
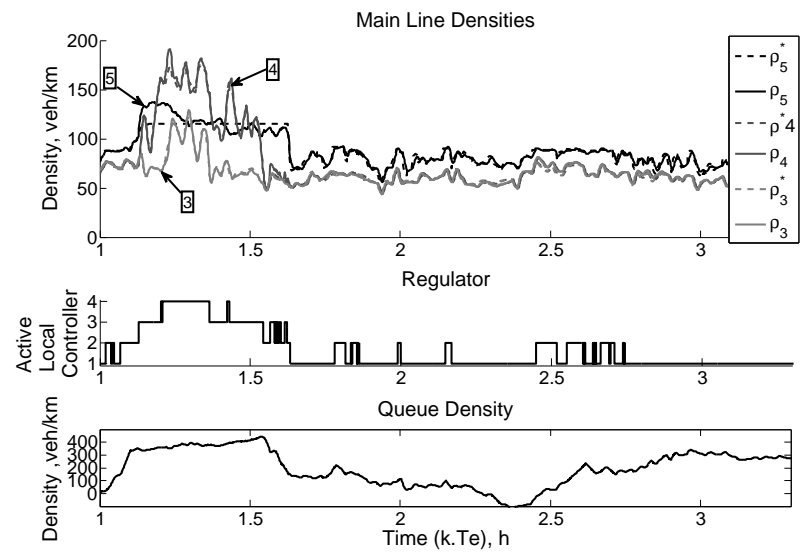

Fig. 16. Local regulation.

Figure 17 shows the evolution of densities subject to the optimal on-ramp flow with local controllers with command saturations. The command saturations guarantee that the 
queue length does not exceed maximum capacity. We also add saturations on the integral term to avoid saturation of the command. Notice that when the queue length reach maximum capacity, the local regulation becomes less effective.
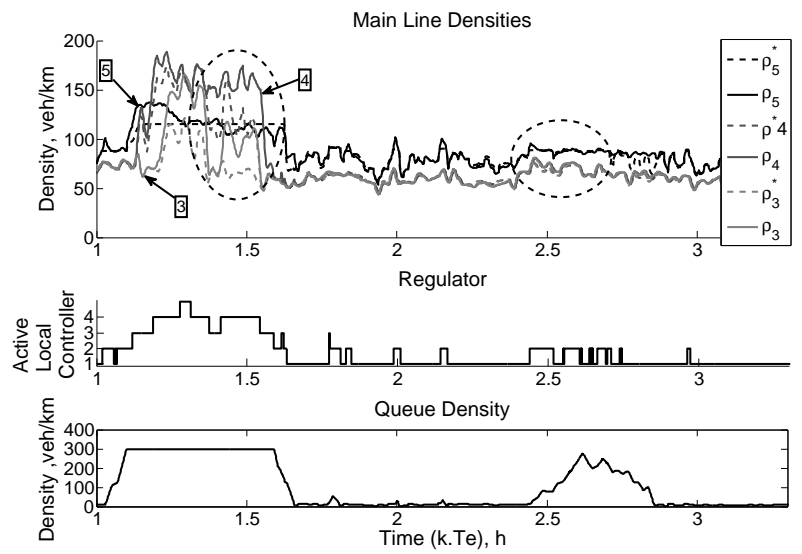

Fig. 17. Local regulation with queue constraints.

These results show the effectiveness of the local controllers to track optimal references face to uncertainties modeled in Section 2.4. The effectiveness of coordinated optimization has already been shown in (Gomes and Horowitz [2006]).

\section{CONCLUSIONS}

In this work we have presented a hierarchical control scheme for traffic control. The proposed approach provides optimal densities and flows references that minimizes the Total Travel Time, the Total Waiting Time and maximized the Total Travel Distance. Local switching PI controllers allow to increase robustness of the controlled system face to parametric uncertainties. These local controllers have be obtained by solving a particular LMI providing stability and pole placement conditions for the modeled switched system. The sensitivity functions between uncertainties and output show the interest of this approach. A real-data based simulation was used to validate the effectiveness of the proposed local controllers where the impact of model un certainties was effectively compensated by the proposed control strategy. As a future work, uncertainties model and uncontrolled dynamics can be used a priori to increase robustness and performance.

\section{REFERENCES}

C. F. Daganzo. The cell-transmission model, part 2: Network traffic. Transportation Research Part B, 29: 79-94, 1995.

C. F. Daganzo. The cell-transmission model, a dynamic representation of highway traffic consistent with the hydrodynamic theory. Transportation Research Part B, 28:269-288, 1994.

N. Geroliminis and C. F. Daganzo. Existence of urbanscale macroscopic fundamental diagram : Some experimental findings. Transportation Research Part B, (42): 759-770, 2008.

G. Gomes and R. Horowitz. Optimal freeway ramp metering using the asymetric cell transmition model. Transportation Research Part C, 14:244-262, 2006.
D Jacquet. Freeway traffic management using linear programming. Proceedings of the 17th IFAC World Congress, 2008.

D Jacquet, J Jaglin, D. Koenig, and C. Canudas De Wit. Non-local feedback ramp metering controller design. Proceedings of the 11th IFAC Symposium on Control in Transportation Systems, 2008.

Dennis Jacquet. Modelisation Macroscopique du Trafic et Controle des Lois de Conservation Non Lineaires Associees. PhD thesis, INP Grenoble, LAG, 2007.

V.F. Montagner, V.J.S. Leite, and P.L.D. Peres. Discretetime switched systems : Pole location and structural constrained control. In Proceedings of the Conference on Decision and Control, pages 6442-6447, Maui, Hawaii USA, December 2003.

L. Munoz, X. Sun, R. Horowitz, and L.Alvarez. Traffic density estimation with the cell transmission model. In Proceedings of the American Control Conference, pages 3750-3755, Denver, colorado USA, June 2003.

L. Munoz, X. Sun, D. Sun, G. Gomes, and R. Horowitz. Methodological calibration of the cell transmission model. In Proceedings of the American Control Conference, pages 798-803, Boston massachusetts, july 2004.

M. Papageorgiou. Some remark on macroscopic traffic flow modelling. 32(16):323-329, 1998.

D. Schrank and T. Lomax. Mobility report. 2007.

Q. Yang and H. N. Koutsopoulos. A microscopic traffic model simulator for evaluation of dynamic traffic management systems. Transportation Research Part B, 4(3): 113-129, 1996. 